\title{
Residual endotoxin induces primary graft dysfunction through ischemia/reperfusion-primed alveolar macrophages
}

\author{
Mahzad Akbarpour, ${ }^{1}$ Emilia Lecuona, ${ }^{1}$ Stephen F. Chiu, ${ }^{1}$ Qiang Wu, ${ }^{1}$ Melissa Querrey, ${ }^{1}$ Ramiro Fernandez, ${ }^{1}$ \\ Félix L. Núñez-Santana, ${ }^{1}$ Haiying Sun, ${ }^{1}$ Sowmya Ravi, ${ }^{1}$ Chitaru Kurihara, ${ }^{1}$ James M. Walter, ${ }^{2}$ Nikita Joshi, ${ }^{2}$ Ziyou Ren, ${ }^{2}$ \\ Scott C. Roberts, ${ }^{3}$ Alan Hauser, ${ }^{2,3}$ Daniel Kreisel, ${ }^{4,5}$ Wenjun Li, ${ }^{4}$ Navdeep S. Chandel, ${ }^{2}$ Alexander V. Misharin, ${ }^{2}$ \\ Thalachallour Mohanakumar, ${ }^{6}$ G.R. Scott Budinger, ${ }^{2}$ and Ankit Bharat ${ }^{1,2}$
}

'Division of Thoracic Surgery, ${ }^{2}$ Division of Pulmonary and Critical Care Medicine, and ${ }^{3}$ Department of Microbiology-Immunology, Feinberg School of Medicine, Northwestern University, Chicago, Illinois, USA ${ }^{4}$ Department of Surgery and ${ }^{5}$ Department of Pathology and Immunology, Washington University in St. Louis, St. Louis, Missouri, USA. ${ }^{6}$ Norton Thoracic Institute, St. Joseph's Hospital and Medical Center, Phoenix, Arizona, USA

\begin{abstract}
Despite the widespread use of antibiotics, bacterial pneumonias in donors strongly predispose to the fatal syndrome of primary graft dysfunction (PCD) following lung transplantation. We report that bacterial endotoxin persists in human donor lungs after pathogen is cleared with antibiotics and is associated with neutrophil infiltration and PGD. In mouse models, depletion of tissue-resident alveolar macrophages (TRAMs) attenuated neutrophil recruitment in response to endotoxin as shown by compartmental staining and intravital imaging. Bone marrow chimeric mice revealed that neutrophils were recruited by TRAM through activation of TLR4 in a MyD88-dependent manner. Intriguingly, low levels of endotoxin, insufficient to cause donor lung injury, promoted TRAM-dependent production of CXCL2, increased neutrophil recruitment, and led to PCD, which was independent of donor NCMs. Reactive oxygen species (ROS) increased in human donor lungs starting from the warm-ischemia phase and were associated with increased transcription and translocation to the plasma membrane of TLR4 in donor TRAMs. Consistently, scavenging ROS or inhibiting their production to prevent TLR4 transcription/translocation or blockade of TLR4 or coreceptor CD14 on donor TRAMs prevented neutrophil recruitment in response to endotoxin and ameliorated PCD. Our studies demonstrate that residual endotoxin after successful treatment of donor bacterial pneumonia promotes PCD through ischemia/reperfusion-primed donor TRAMs.
\end{abstract}

\section{Introduction}

Prolonged mechanical ventilation, aspiration of gastric contents into the lungs around the time of death, and immune dysfunction after brain death all contribute to increased rates of bacterial infections in donor lungs $(1,2)$. Despite the routine use of bronchoalveolar lavage culture and empiric and pathogen-directed antimicrobial therapy, the presence of bacteria in donor lungs is associated with increased risk for primary graft dysfunction (PGD), the predominant cause of mortality following lung transplantation (3). These high rates of bacterial recovery and the associated PGD following transplant are major reasons why donor lungs have a utilization rate of only about $15 \%$, which is one of the lowest among all solid organs (www.unos.org). The low utilization of donor lungs accounts for the high mortality and long wait times observed in patients requiring lung transplantation. How bacteria in the donor lungs predispose to the pathogenesis of PGD and why antimicrobial therapy is unable to prevent lung allograft injury remains unclear.

Conflict of interest: The authors have declared that no conflict of interest exists. Copyright: ( 2020 , American Society for Clinical Investigation.

Submitted: December 20, 2019; Accepted: May 13, 2020; Published: July 20, 2020

Reference information: J Clin Invest. 2020;130(8):4456-4469.

https://doi.org/10.1172/JCl135838.
Host neutrophils recruited to the lung allograft mediate lung ischemia/reperfusion injury leading to PGD, the most important risk factor for early and chronic allograft failure, but are also necessary for the clearance of bacterial and fungal pathogens (4-13). Since neutrophil depletion is unlikely to be tolerated in immunosuppressed lung transplant patients, it is critical to delineate mechanisms leading to neutrophil recruitment immediately after transplant. The severity of neutrophil recruitment and the resulting PGD varies dramatically among lung transplant recipients. Because broad-spectrum antibiotics targeting most Gramnegative organisms are routinely administered to both the donor and recipient before transplantation, the role of bacteria in the donor lung in driving this heterogeneity has been overlooked.

Surprisingly, we detected lipopolysaccharide (LPS), also referred to as endotoxin, in human donor lungs with bacterial pathogens before transplant, despite antibiotic therapy. LPS is a prototypical pathogen-associated molecular pattern (PAMP) in the outer cytoplasmic membrane of Gram-negative bacteria that signals through TLR4 receptors to induce neutrophil influx into the alveolar space $(4,14)$. We recently discovered that donor origin pulmonary intravascular nonclassical monocytes (NCMs) trigger neutrophil recruitment following lung transplantation through the production of neutrophil chemoattractant CXCL2 $(11,13,15)$. The donor NCMs also recruit spleen-derived CCR2 ${ }^{+}$classical mono- 
Human LTX

A

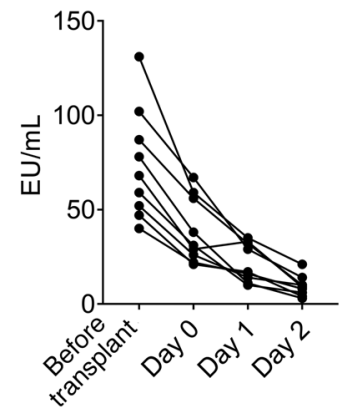

B $\square$ Before reperfusion $\square$ After reperfusion

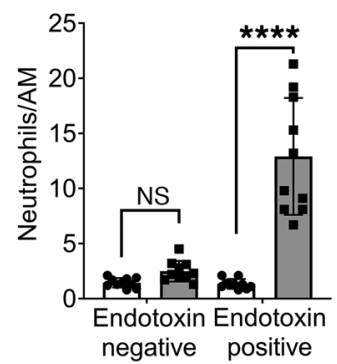

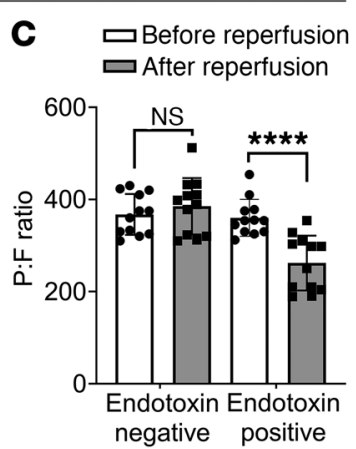

Murine LTX

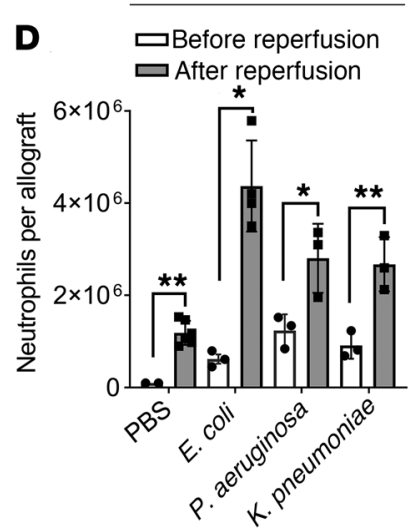

Figure 1. Persistence of endotoxin in human and murine donor lungs is associated with neutrophil influx and PGD following transplantation. (A) Human donor lungs with microbial cultures positive for Gram-negative bacteria were treated with antimicrobials, resulting in clearance of the pathogen at the time of transplantation. To test for persistence of endotoxin, BALF serially obtained from the same patient was analyzed ( $n=9$ ). (B) Human donor lungs from endotoxin-negative and endotoxin-positive lungs were analyzed for tissue neutrophil infiltration before reperfusion and 30 minutes after reperfusion using intraoperative lung biopsy of the inferior lingular segment $(n=10)$. (C) To determine the function of the lung allograft, we obtained blood directly from the pulmonary vein confluence immediately proximal to the site of venous anastomosis and analyzed partial pressure of oxygen $\left(\mathrm{PaO}_{2}\right.$ $\mathrm{mmHg}$ ) before and after reperfusion. The $\mathrm{PaO}_{2}$ to fractional inspired oxygen $\left(\mathrm{FiO}_{2}\right)$ ratio $(\mathrm{P} / \mathrm{F}$ ratio) was then calculated for lungs negative and positive for endotoxin $(n=12)$. (D) Donor mice were administered a low dose of LPS i.t. (E. coli [0.5 $\mu \mathrm{g} / \mathrm{g} \mathrm{BW],} \mathrm{P.} \mathrm{aeruginosa} \mathrm{[0.2} \mu \mathrm{g} / \mathrm{g}$ BW], and K. pneumoniae [0.2 $\mu \mathrm{g} / \mathrm{g} \mathrm{BW]}$ ), insufficient to cause injury in native lung, and then lungs were transplanted into allogeneic recipients. The neutrophil infiltration was then analyzed following transplant $(n=3-6)$. Graphs show mean \pm SD. Graphs were analyzed by unpaired $t$ test. ${ }^{*} P<0.05 ;{ }^{* *} P<0.01 ;{ }^{* * *} P<0.0001$.

cytes (CMs) to the allograft, which contribute to neutrophil extravasation, worsening lung injury (12). Thus, both donor-derived NCMs and recipient CMs play complementary roles in neutrophil recruitment and extravasation to initiate lung transplant ischemia/ reperfusion injury. These mechanisms differ from observations in naive mice treated with LPS in which tissue-resident alveolar macrophages (TRAMs), or other cell populations in the lung, may be necessary for neutrophil recruitment (16-19). We therefore hypothesized that LPS from the different bacterial pathogens and perhaps other PAMPs, persisting after successful antimicrobial therapy, contribute to excessive neutrophil recruitment after lung transplantation by augmenting signaling through NCMs or CMs, and/or activated parallel pathways through TRAMs.

We found that endotoxin can persist in human donor lungs despite pathogen clearance and is associated with increased neutrophil infiltration and PGD after lung transplantation. Pharmacologic and genetic depletion of donor NCMs attenuated neutrophil recruitment following transplant of naive donor lungs $(12,13)$, but did not abrogate neutrophil recruitment and PGD in donor lungs containing endotoxin. In addition, exposure of donor TRAMs to ischemia/reperfusion primed them to release CXCL2 in response to endotoxin through a TLR4/ MyD88 pathway, obviating the requirement for donor NCMs in neutrophil recruitment to the allograft. Donor lungs produced reactive oxygen species (ROS), beginning in the warm-ischemia phase, which led to both an increase in TLR4 transcription and its translocation to the cell plasma membrane. Accordingly, ROS scavengers, blockade of TLR4, or TLR4 coreceptor CD14 resulted in amelioration of PGD when endotoxin was present in the donor lungs. Our results suggest that a multipronged strategy that targets both TRAMs and NCMs within the donor lung can reduce the severity of PGD in donors with a history of bacterial pneumonia or colonization.

\section{Results}

Endotoxin persists in donor lung despite pathogen clearance, and predisposes to PGD. Recruitment of host neutrophils to the lung allograft following lung transplantation mediates lung ischemia/ reperfusion injury (10). To determine whether bacterial split products in the donor lung could exacerbate neutrophil influx after transplant, we analyzed human donor lungs that were positive for Gram-negative bacteria before transplant but treated with antimicrobials. These human donor lungs had normal $\mathrm{P} / \mathrm{F}$ ratio in the donor before transplant and no pulmonary infiltrates on chest radiograph or computed tomography. Successful treatment of the infection was evident by negative microbial cultures of bronchoalveolar fluid (BALF) collected at the time of transplantation. However, as shown in Figure 1A, the BALF of successfully treated human donor lungs obtained at transplant revealed residual endotoxin that persisted following transplantation. Intriguingly, following transplantation, these endotoxin-containing donor lungs showed physiological markers of injury, including significantly higher neutrophil infiltration as well as reduced $\mathrm{P} / \mathrm{F}$ ratio after transplant, compared with those without endotoxin (Figure 1, B and $\mathrm{C}$ ), although there was no significant difference in the neutrophil count and $\mathrm{P} / \mathrm{F}$ ratio between the 2 groups in the donor before lung procurement (Figure 1, B and C). We replicated these observations in a murine model of lung transplantation by administering intratracheal (i.t.) PBS control or a low dose of LPS from 3 different bacteria $(0.5 \mu \mathrm{g} / \mathrm{g}$ BW E. coli, $0.2 \mu \mathrm{g} / \mathrm{g}$ BW P. aeruginosa or $0.2 \mu \mathrm{g} / \mathrm{g}$ BW K. pneumoniae) to donor mice before transplantation into allogeneic murine recipients. The low dose was insufficient to cause neutrophil infiltration or injury in the native lungs. However, the grafts containing LPS experienced severe PGD at 24 hours, which was associated with an increase in chemokines in the BALF (Supplemental Figure 1; supplemental material available online with this article; https://doi.org/10.1172/JCI135838DS1), includ- 
A

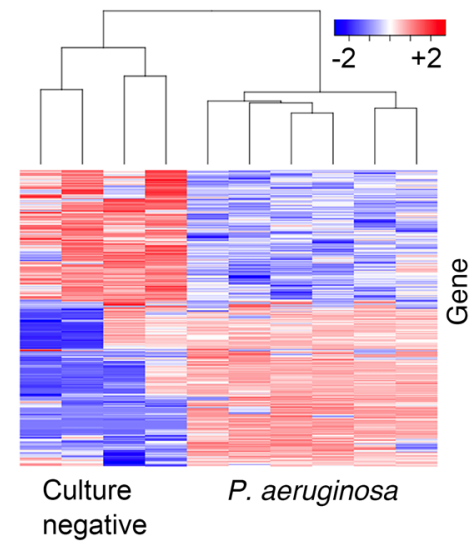

C

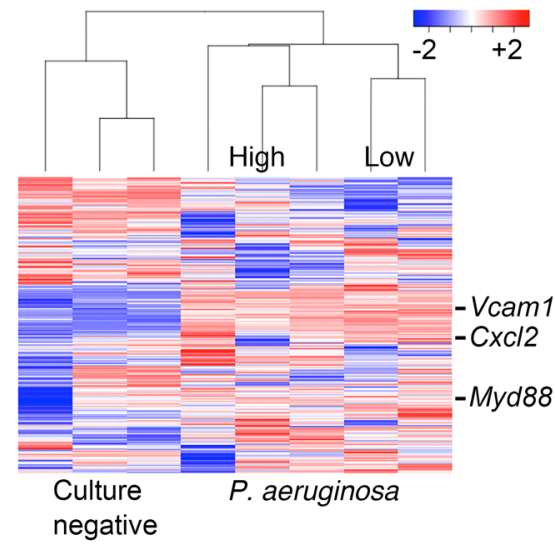

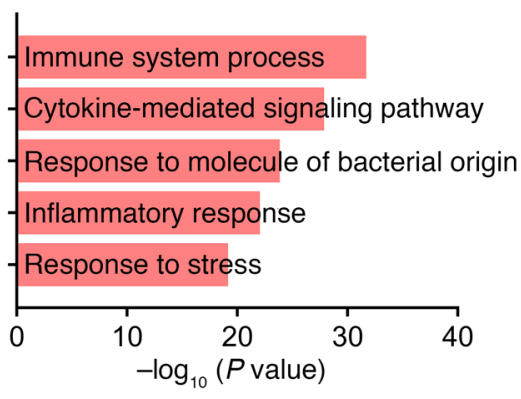

D

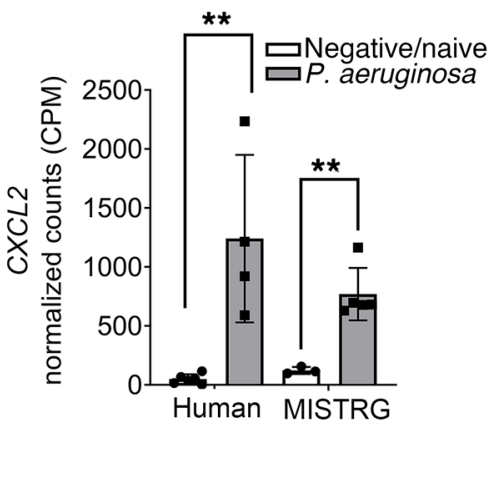

Figure 2. TRAMs upregulate CXCL2 in response to endotoxin and pseudomonas. Transcriptomic profiling of human TRAMs during Gram-negative bacterial pneumonia revealed upregulation of CXCL2, a known neutrophil chemoattractant. TRAMs were identified as CD15-HLA-DR+CD169+ $\mathrm{CD}^{2} \mathrm{C}^{++}$, and flow sorted for RNA isolation, library preparation, and sequencing (culturenegative, $n=4$; $P$. aeruginosa-positive, $n=6$ ). (A) Heatmap of top 1000 most significantly differentially expressed genes ranked by FDR $q$ value. Both samples and genes are grouped by hierarchical clustering. (B) Functional enrichment analysis with GO biological processes was performed with GOrilla with the top 1000 genes upregulated in P. aeruginosa-positive samples for all clusters. In total, 443 significantly upregulated genes were used to identify the top 5 biological processes $(-\log 10$ [ $P$ value]). (C) Heatmap of pathway genes (750 genes) based on the top $\mathrm{GO}$ processes identified in B (С०:0002376, ६0:0019221, ५O:0002237, ६0:00 06954,G0:0006950). Hierarchical clustering shows the expression of these genes in naive, low, and high virulence samples for MISTRG mice. Selective genes are highlighted $(n=3-4)$. (D) Normalized CPM counts of CXCL2 in both human and MISTRG mouse following infection with $P$. aeruginosa $(n=$ 3-6). Graph show mean $\pm S D$ and was analyzed by unpaired $t$ test. ${ }^{* *} P<0.01$. ing CXCL2, a potent neutrophil chemoattractant $(13,20)$, as well as profound neutrophil influx (Figure 1D). These data suggested that low levels of endotoxin may not cause clinically significant lung injury; however, following ischemia/reperfusion, the susceptibility of lung allografts to residual endotoxin increases substantially. Finally, recent studies have suggested that the donor microbiome may play a role in the development of lung allograft rejection (21-24). Since the role of the donor lung microbiome in the pathogenesis of PGD is uncertain, we analyzed the BALF from human donor lungs by 16S PCR high-throughput screening, as previously described $(25,26)$. BAL samples were distinct from rectal samples $(R=0.977, P=0.002)$, and no microbial clustering within BAL samples was apparent. PGD sample (patient 9 ) was not dissimilar from non-PGD samples (patients $1-7,8,10)(R=-0.279, P=0.625)$. There was also no variation in the donor lung microbiome with regard to endotoxin-containing organisms and this lack of variation is, therefore, unlikely to explain our results (Supplemental Figure 2).

CXCL2 secreted from TRAMs contributes to neutrophil recruitment and lung injury following bacterial infection or LPS administration. To determine the mechanisms by which endotoxin in donor lungs accentuates PGD, we first studied the mechanism of endotoxin-induced lung injury in the native mouse lung. Given that LPS from all 3 bacterial species induced PGD and neutrophil influx, for subsequent studies we used LPS from E. coli since it has been used most commonly in published literature. Consistent with prior reports, we found that 24 hours after high dose $(2 \mu \mathrm{g} / \mathrm{g}$
BW) i.t. LPS administration there was a robust neutrophil influx (Supplemental Figure 3A) in native lungs with more than $80 \%$ of neutrophil extravasated into the alveolar space (Supplemental Figure 3B). This was accompanied by pulmonary edema and histologic signs of injury (Supplemental Figure 3, C and D) $(27,28)$. We then performed RNA-Seq analysis of flow-sorted TRAMs isolated from BALF in patients with severe pneumonia resulting in respiratory failure in our medical intensive care unit (16). Specifically, we compared alveolar macrophage transcriptomes from patients with confirmed pneumonia secondary to Pseudomonas aeruginosa (based on quantitative culture of the same BALF sample) to transcriptomes of patients with negative quantitative culture and found that differentially expressed genes were associated with gene ontology (GO) processes involved in immune activation (Figure 2, A and B). We found that those genes associated with the GO processes in human subjects were also differentially expressed in the TRAMs of humanized $\mathrm{Rag}^{-/-} \mathrm{IL}_{2 \mathrm{~g}^{-/}}$mice with transgenes for human GM-CSF and IL-3 (MISTRG mice) infected with Pseudomonas (Figure 2C). Furthermore, compared with those without pneumonia, TRAMs from patients with Pseudomonas pneumonia and MISTRG mice infected with $P$. aeruginosa demonstrated a significant increase in the expression of the primary neutrophil chemokine, CXCL2 (Figure 2D), which we have shown to be pivotal in neutrophil chemotaxis to the reperfused lung allograft $(11,13,15)$.

To further investigate whether TRAMs were responsible for CXCL2 production in response to LPS in TLR4-dependent manner, 
A

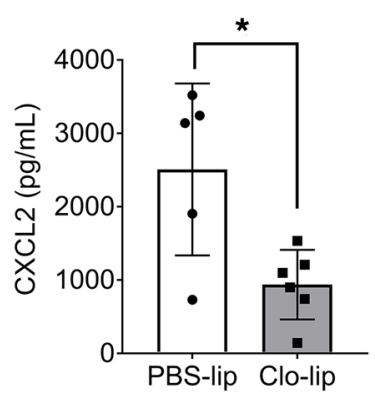

D

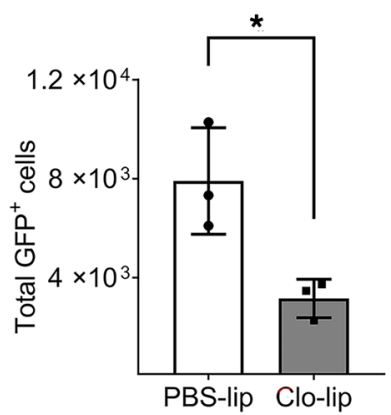

B

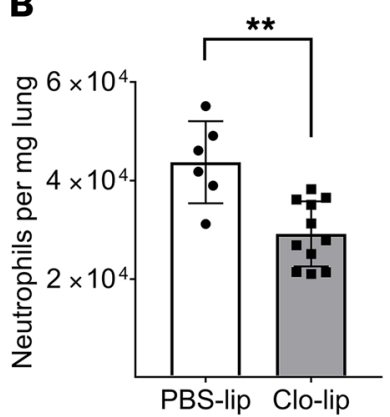

$\mathbf{E}$

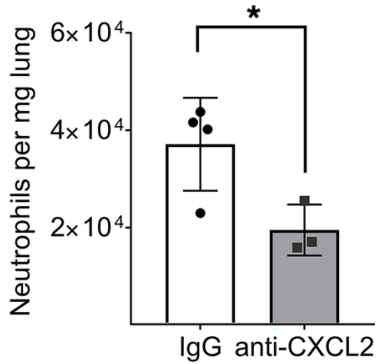

C
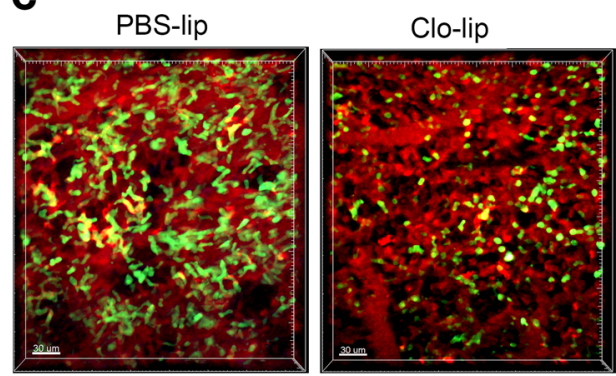

$\mathbf{F}$

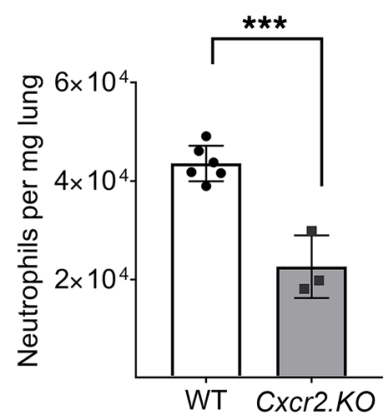

Figure 3. Influx of neutrophils into LPS-treated lung is abrogated by selective depletion of TRAMs. (A) CXCL2 chemokine levels in BALF of LPS-treated mice injected with PBS-lip or Clo-lip i.t. $(n=5-6)$. (B) Quantification of neutrophil infiltration into lungs in TRAM-depleted compared with PBS-lip control mice 24 hours after LPS administration $(n=6-11)$. Neutrophils were gated as live CD45+Ly6C+CD11 ${ }^{+}$CD24 ${ }^{+}$SSC ${ }^{\text {hi }}$ cells. (C) Representative intravital 2-photon microscopy images of control PBS-lip- or Clo-lip-treated lungs 4 hours after LPS administration in B6 LysM-GFP mice (also refer to Supplemental Videos 1 and 2). Q-dots (655-nm nontargeted) were injected 5 minutes before imaging to label the vessels red $(n=3)$. (D) Quantification of total GFP ${ }^{+}$neutrophils from C $(n=3)$. (E) Quantification of neutrophil infiltration into lungs in LPS-treated mice previously injected with IgC or an antibody against $C X C L 2$ ( $n=3-4)$. (F) Quantification of neutrophil infiltration into lungs in LPS-treated mice in WT (WT) compared with Cxcr2 ${ }^{-1-}($ Cxcr2.KO) mice ( $n=3-6)$. Scale bar: $30 \mu \mathrm{m}$. All graphs show mean $\pm \mathrm{SD}$. Graphs were analyzed by unpaired $t$ test. ${ }^{*} P<0.05 ;{ }^{* *} P<0.01 ;{ }^{* *} P<0.001$.

we analyzed expression of TLR4 in previously published singlecell RNA-seq data sets generated from nondiseased human lungs (29-31) (Supplemental Figure 4). Expression of TLR4 was largely restricted to FABP4-positive TRAMs, although some endothelial cells and monocytes also demonstrated expression of TLR4. Given that endothelial cells face the vasculature and monocytes in human lungs are largely retained in the blood vessels $(13,32)$, TRAMs are likely to be the predominant secretors of CXCL2 in response to TLR 4 stimulation by LPS present in the alveolar space. Nevertheless, to confirm that TRAMs drive neutrophil recruitment in response to LPS, we administered clodronate-loaded liposomes intratracheally to selectively deplete TRAMs in WT mice (Supplemental Figure 5). Depletion of TRAMs before LPS challenge in WT mice resulted in a significant decrease in CXCL2 levels in BALF (Figure 3A) along with a significant reduction in neutrophil infiltration in lung tissue, determined by flow cytometry (Figure 3B) and 2-photon microscopy (Figure 3, C and D; and Supplemental Videos 1 and 2). Next, to determine whether CXCL2 signaling was necessary for neutrophil recruitment in response to LPS, we treated mice with anti-CXCL2 antibodies (13). Neutralization of CXCL2 resulted in a significant attenuation of neutrophil recruitment to the injured lungs, 24 hours after LPS administration (Figure 3E). Similarly, mice deficient in the CXCL2 receptor $\left(\mathrm{Cxcr2} 2^{--}\right)$did not develop neutrophil influx in response to i.t. LPS (Figure 3F). Moreover, depletion of circulating CMs using anti-
CCR2 antibody (Supplemental Figure 6, A-C), NCMs using intravenously administered Clo-lip (Supplemental Figure 6, D-F), or simultaneous depletion of monocytes, peribronchial interstitial macrophages, and lung CD11 $\mathrm{b}^{+}$dendritic cells using CD11b-DTR system, had no effect on neutrophil trafficking in response to i.t. LPS (Supplemental Figure 6, G and H). Taken together, these data demonstrate that TRAMs are responsible for neutrophil influx in response to LPS through the production of CXCL2.

Donor TRAMs exacerbate PGD in donor lung containing endotoxin through the production of CXCL2. We have recently shown that donor origin pulmonary intravascular NCMs recruit neutrophils following transplant and contribute to the pathogenesis of PGD in a mouse model of lung transplantation $(12,13)$. As bacterial infections are common in human donor lungs, we investigated whether NCMs, TRAMs, or both would mediate neutrophil recruitment in donor lungs exposed to LPS. Accordingly, we treated donors with a low dose of LPS $(0.5 \mu \mathrm{g} / \mathrm{g} \mathrm{BW})$, insufficient to cause donor lung injury, and found that while LPS-free donor lungs from mice lacking NCMs $\left(\mathrm{Nr}_{\mathrm{ar}} \mathrm{1}^{--}\right)$did not develop neutrophil recruitment and PGD following lung transplantation (13), in contrast, donor lungs from low-dose LPS-treated $\mathrm{Nr}_{4} \mathrm{a1}^{-/-}$mice did (Figure 4, A and B). Similarly, pharmacological depletion of donor NCMs using i.v. Clo-lip prevented neutrophil recruitment following murine lung transplant of LPS-free (13), but not of LPS-containing, donor lungs (Figure 4, A and B), suggesting that neutrophil recruitment associ- 
A

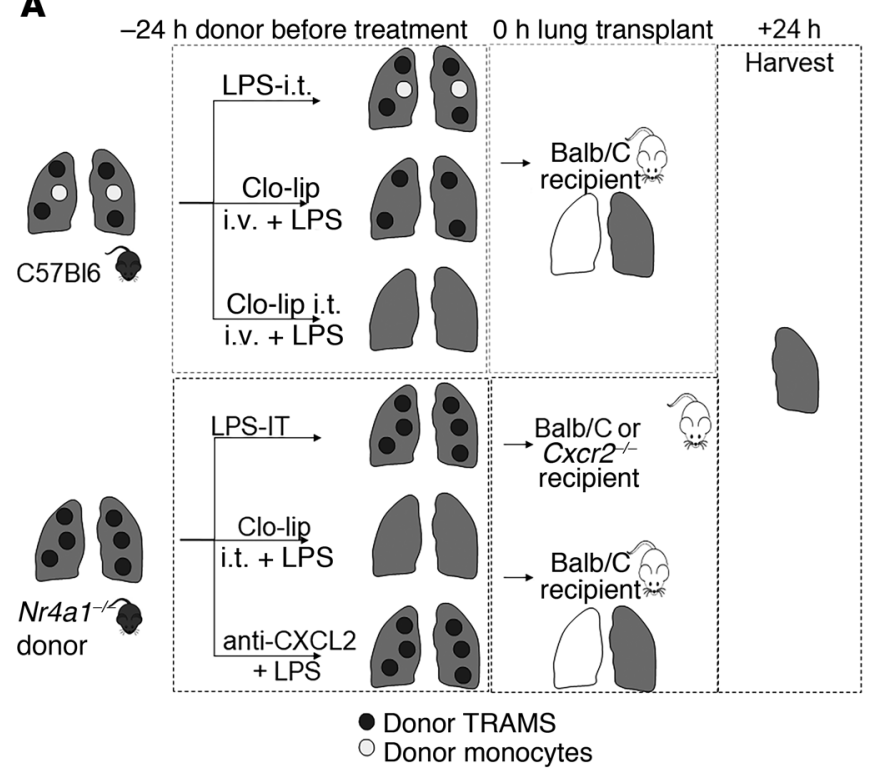

B

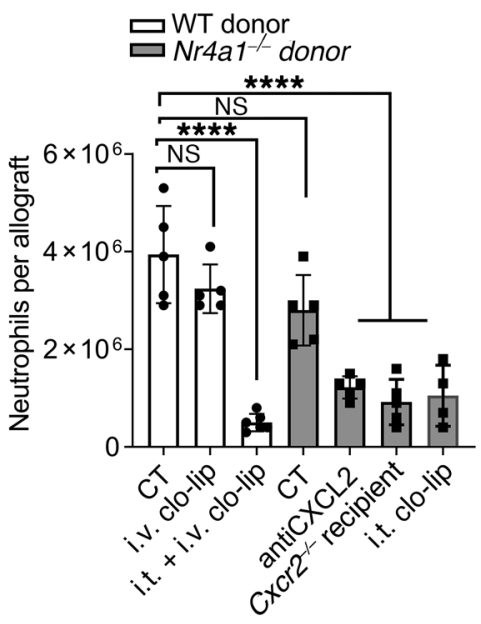

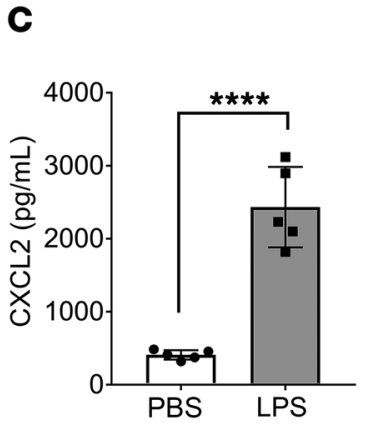

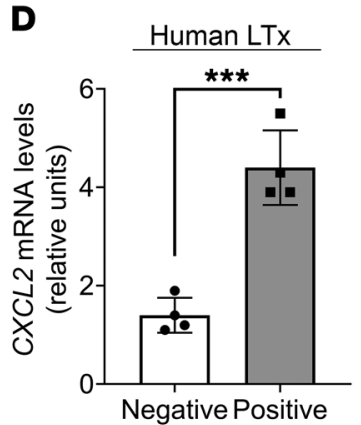

Figure 4. Influx of recipient neutrophils into the allograft containing LPS is abrogated by depletion of donor TRAMs and is CXCL2 dependent. (A) Diagram depicting experiment shown in B. WT C57BL/6 mice received BALB/c allogeneic donor lungs containing low-dose LPS (0.5 $\mu$ g/g BW) injected i.t., insufficient to cause native lung injury. To deplete NCMs, donors were treated with i.v. Clo-lip before i.t. LPS. To deplete both donor monocytes and donor TRAMs, we treated the donors with i.v. Clo-lip and i.t. Clo-lip, respectively, before i.t. LPS. Next, we used Nr4a1/- donor lungs containing low-dose LPS and transplanted them into WT recipients after depletion of TRAMs using i.t. Clo-lip, or using PBS-lip as control. Last, to determine whether CXCL2 secretion by donor TRAMs led to neutrophil recruitment, we treated recipients of $\mathrm{Nr}_{4} \mathrm{a} 1^{-/-}$donor lungs containing low-dose LPS with anti-CXCL2 antibodies at the time of transplantation. Additionally, we transplanted $\mathrm{Nr}_{4 a 1^{-/-}}$donor lungs containing low-dose LPS into $\mathrm{Cxcr2}^{-/-}$recipients and determined neutrophil infiltration at 24 hours.(B) Quantification of neutrophil infiltration into lungs after these treatments was performed using flow cytometry ( $n=4-5)$. (C) CXCL2 chemokine levels in BALF of recipient mice after receiving low-dose LPS-treated $\mathrm{Nr}_{4} \mathrm{a}^{-1-}$ donor lungs compared with PBS-treated $\mathrm{Nr} 4 a 1^{-/-}$donor lungs $(n=5)$. (D) TRAMs were isolated from endotoxin-negative and endotoxin-positive human donor lungs at 120 minutes after reperfusion and analyzed for CXCL2 transcription $(n=4)$. All graphs show mean \pm SD. Graph in B was analyzed by 1-way ANOVA followed by Tukey's post hoc test. Graphs in C and $\mathbf{D}$ were analyzed by unpaired $t$ test. ${ }^{* *} P<0.01 ;{ }^{* * *} P<0.0001$. CT, control.

ated with LPS in donor lungs is NCM independent. On the other hand, depletion of TRAMs in LPS-treated $\mathrm{Nr} \mathrm{al}^{-/}$donor lungs before transplant using i.t. Clo-lip or depletion of both TRAMs and NCMs by i.t. plus i.v. Clo-lip in WT donor lungs attenuated the LPS-induced neutrophil infiltration (Figure 4, A and B).

We have reported that $\mathrm{Nr}_{4 a 1^{--}}$donor lungs have reduced CXCL2 levels following transplant when compared with WT donor lungs (13). However, administration of low-dose LPS to $\mathrm{Nr}^{4} \mathrm{al}^{-1-}$ donor lungs before transplantation resulted in increased CXCL2 levels (Figure 4C). We also found increased CXCL2 mRNA expression in TRAMs isolated from human donor lungs positive for endotoxin compared with those negative for endotoxin following transplantation reperfusion (Figure 4D). To determine whether CXCL2 was necessary for the recruitment of neutrophils in endotoxin- containing donor lungs, we treated recipients with an anti-CXCL2 antibody immediately after transplantation or used $\mathrm{Cxcr}^{-/-}$mice as transplant recipients. In both cases, neutrophil recruitment after transplantation of the endotoxin-containing donor lung was attenuated (Figure 4, A and B). Collectively, these results show that ischemia/reperfusion-primed TRAMs can recruit neutrophils in lung allografts containing low levels of endotoxin through CXCL2 release, obviating the requirement for NCMs.

TRAM-mediated neutrophil recruitment in response to endotoxin is dependent on TLR4-MyD88 signaling. The pattern recognition receptor for LPS is toll-receptor 4 (TLR4), which interacts with the intracellular adaptor protein MyD88 to enable signal transduction (33). In order to test whether the TLR4-MyD88 signaling pathway was responsible for the LPS priming of TRAMs before lung trans- 

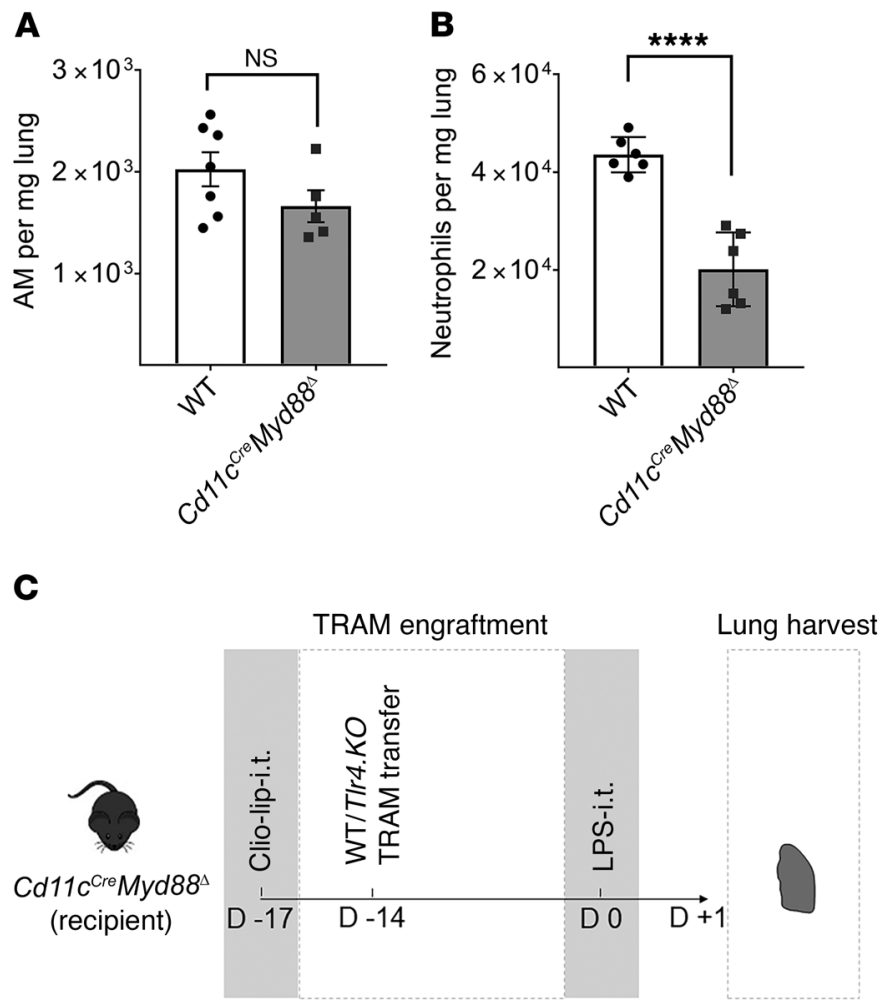

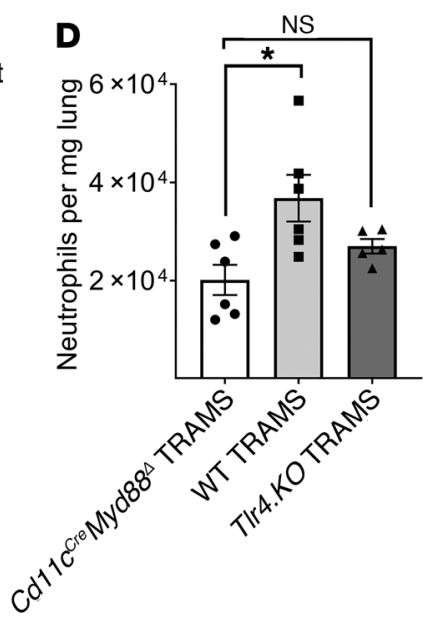

CXCL2 upon stimulation with LPS (Figure 6, C and D). We next performed RNA-Seq analysis of donor TRAMs experiencing ischemia/reperfusion injury $(13,34)$, which revealed upregulation of TLR4 and its coreceptor CD14 (Figure 7A). We validated these findings by performing quantitative real-time PCR of TRAMs isolated from murine donor lungs before and after ischemia (Figure 7B). To understand TLR4 regulation during ischemia/ reperfusion injury, we performed a time course after ischemia, analyzing both Tlr4 mRNA levels and protein expression at the plasma membrane in TRAMs. We found that Tlr4 mRNA levels started increasing 4 hours after ischemia, reaching significance at 24 hours (Figure 7C). Using flow cytometry, we determined that TLR4 protein expression at the plasma membrane reached statistical significance at 4 hours after ischemia, keeping a constant expression at 24 hours (Figure 7D). Moreover, immunocytochemistry (ICC) performed in donor TRAMs isolated at serial time points following transplantation showed that TLR4 increasingly accumulated toward the cell periphery following transplantation (Figure 7E, Supplemental Figure 8 and Supplemental Figure 9). These results suggest that ischemia/reperfusion injury induces TLR4 translocation to the plasma membrane at earlier time points (35), followed by increased de novo synthesis at later time points. Finally, to determine whether TLR4 signaling is necessary for LPS-induced activation of donor lung TRAMs, we administered LPS in the presence of TLR4 antagonist (TAK-242) or CD14/TLR4 antagonist (IAXO-101) and then transplanted these lungs into WT recipients. We found that anti-TLR4 treat- using murine models. Similar to humans, TRAMs isolated from
murine donor lungs after ischemia produced significantly more 


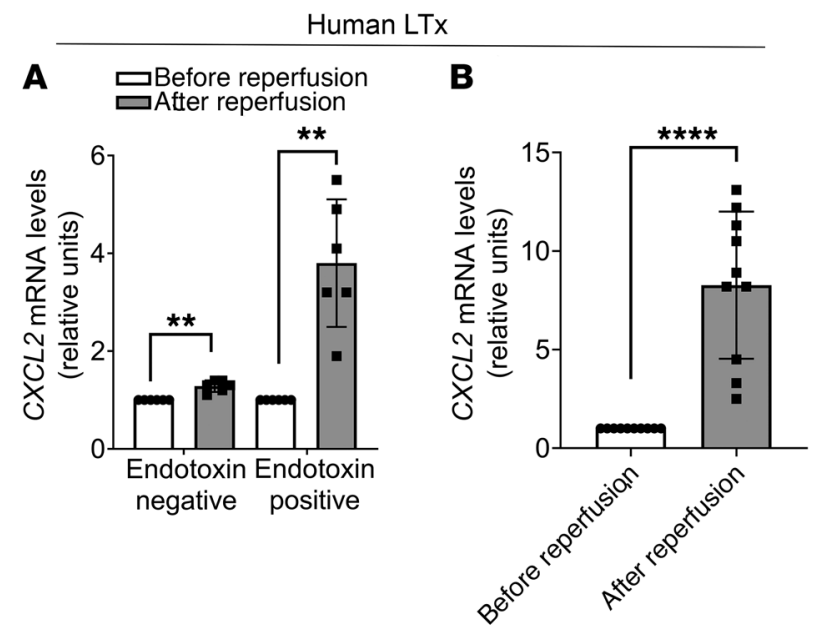

C
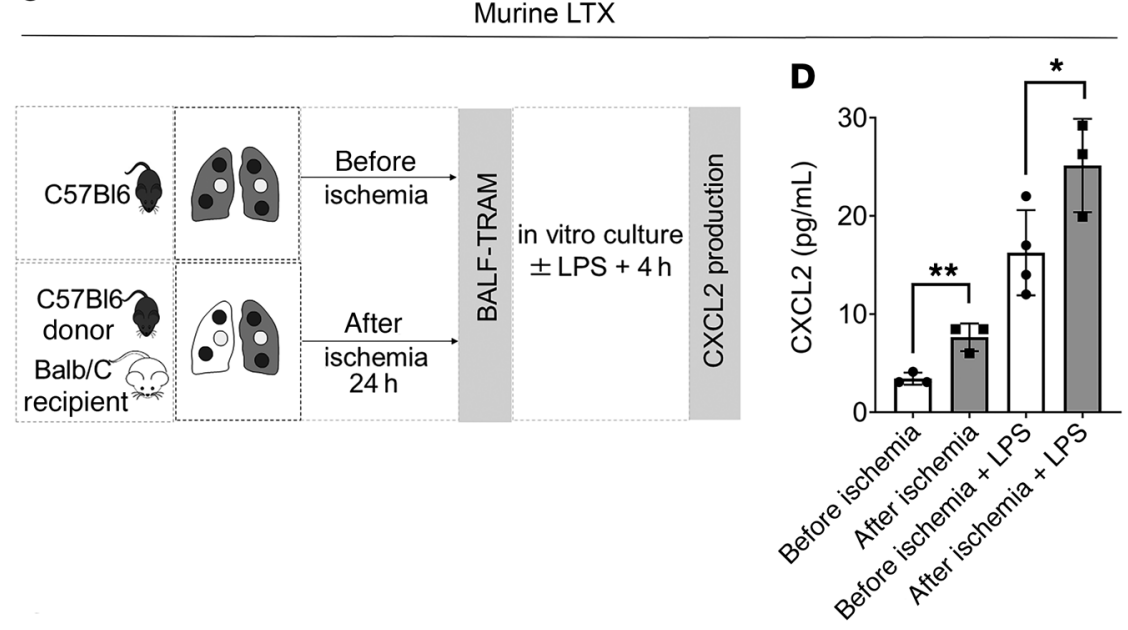

Figure 6. Ischemia/reperfusion-primed TRAMs have augmented response to endotoxin. (A) Relative CXCL2 mRNA levels of human TRAMs from endotoxin-negative and endotoxin-positive patients before and after reperfusion $(n=6)$. (B) Fresh TRAMs from endotoxin-negative human donor lungs were isolated before transplantation or 120 minutes after reperfusion and then stimulated with endotoxin in vitro $(0.01 \mu \mathrm{g} / \mathrm{mL}$ LPS $)(n=10)$. (C) Diagram depicting the experiments illustrated in $\mathbf{D}$, where TRAMs harvested before and after ischemia were challenged with LPS in vitro. (D) CXCL2 chemokine levels in the supernatant of murine TRAMs harvested before and after ischemia and challenged in vitro with LPS $(0.01 \mu \mathrm{g} / \mathrm{mL})$ for 4 hours $(n=3-4)$. (A-C) Graphs were analyzed by unpaired $t$ test. ${ }^{*} P<0.05 ;{ }^{* *} P<0.01 ;{ }^{* * *} P<0.0001$.

ment suppressed the neutrophil influx after transplant associated with LPS (Figure 7F).

ROS released during warm ischemia and following reperfusion increased the transcription of TLR4 and its translocation to the cell membrane in TRAMs. ROS generated in the mitochondria have been implicated in signaling pathways activated after ischemia/ reperfusion injury $(36,37)$. To explore whether ROS are necessary for the increased transcription of TLR 4 in donor TRAMs, we analyzed ROS generation in donor human lung tissue and TLR4 mRNA expression in TRAMs at 5 consecutive time points during ischemia/reperfusion. Donor tissue was harvested as follows: (a) in situ native lung: biopsies obtained after opening donor's chest cavity and before procurement; (b) end of cold ischemia: biopsies obtained after the donor lung was removed from cold-storage but before initiation of implantation; (c) end of warm ischemia: biopsies obtained from the donor lung that sustained warm ischemia during implantation but immediately before reperfusion; (d) 30 minutes after reperfusion; and (e) 120 minutes after reperfusion. We found that ROS increased during the warm-ischemia phase and continued to increase at 30 and 120 minutes after reperfusion (Figure 8A). Intriguingly, TLR 4 transcription in human donor TRAMs did not increase until 30 minutes after reperfusion but continued to increase at the 120-minute time point (Figure $8 \mathrm{~A}$ ). It was clinically infeasible to evaluate TRAMs from the donor lung tissue site at longer time points since the chest of the recipient is closed at the conclusion of the surgical procedure. Accordingly, we performed experiments using exogenous ROS to replicate these findings. We cultured murine TRAMs in the presence of $100 \mu \mathrm{M}$ hydrogen peroxide, as previously described (35). At 1 hour, we did not observe any change in Tlr4 transcription, but its translocation to the cell membrane increased significantly (Supplemental Figure 10, A and B). Unfortunately, we could not evaluate the effects of hydrogen peroxide on TRAMs in vitro beyond 1 hour, as we observed progressive cell death at longer time points (Supplemental Figure 10C). Nevertheless, using the murine model of lung transplantation, we could confirm a role for mitochondrial ROS by using the general ROS scavenger N-acetylcysteine (NAC) (38), and the mitochondrial ROS scavengers Mito-Tempo (MT) (39) and dimethyl malonate (DM) (40) to abrogate the ischemia/reperfusion-induced increase in Tlr4 transcription and expression at the plasma membrane. Indeed, donor TRAMs did not develop an increase in Tlr4 transcription or TLR4 protein translocation to the plasma membrane following murine lung transplantation when treated with NAC, MT, or DM as demonstrated by both qualitative and quantitative measures (Figure 8, B-D). Moreover, NAC treatment prevented neutrophil recruitment and development of PGD in endotoxin-containing $\mathrm{Nr}_{4 a 1^{-/}}$donor lungs (Figure 8, E and F).

\section{Discussion}

Despite the increasing numbers of lung transplants worldwide, there is a significant gap between the demand and availability of lungs for transplants (41). Many potential donor lungs with adequately treated donor pneumonias are turned down at the time of evaluation because of concerns for the development of PGD following transplantation (42-44). While neutrophil recruitment into the transplanted lung allograft is essential for the development of PGD, a lack of insight into mechanisms that drive neutrophils 
A

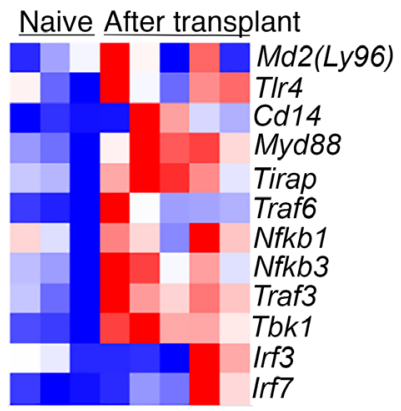

Min $\quad$ Max
B

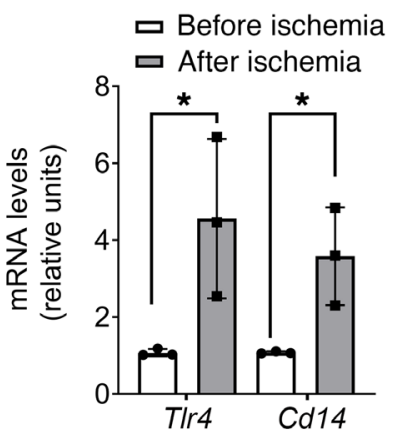

C

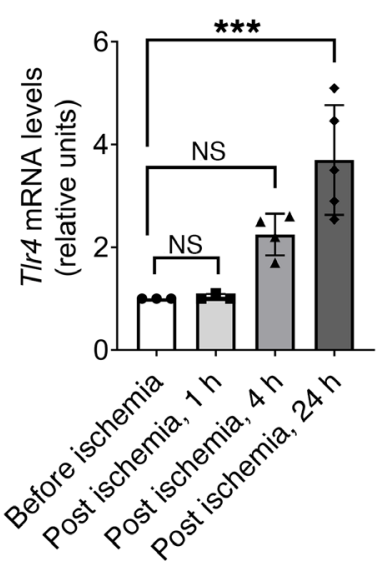

D

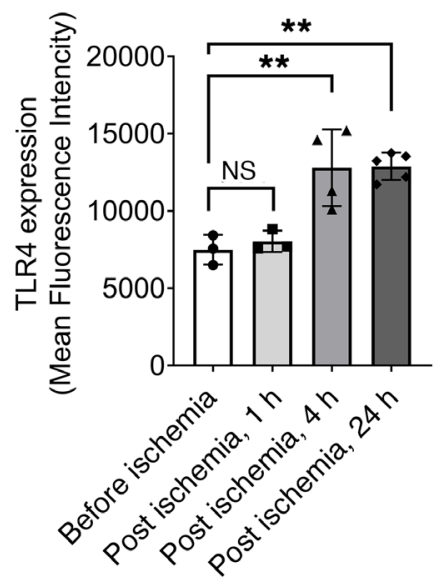

E

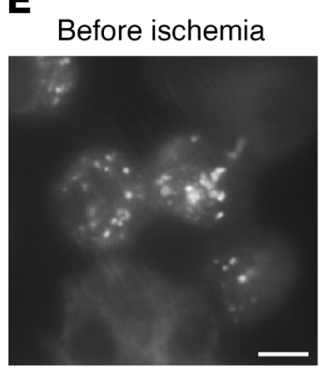

After ischemia, $1 \mathrm{~h}$

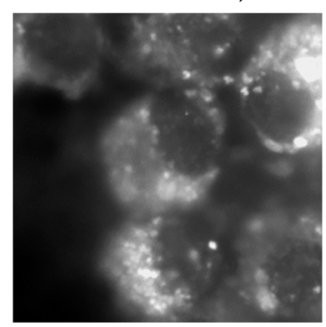

After ischemia, $4 \mathrm{~h}$

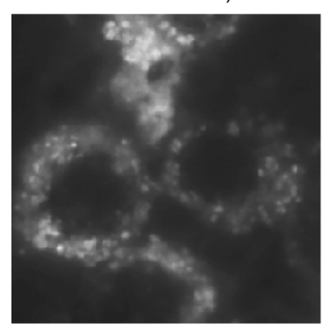

After ischemia, $24 \mathrm{~h}$

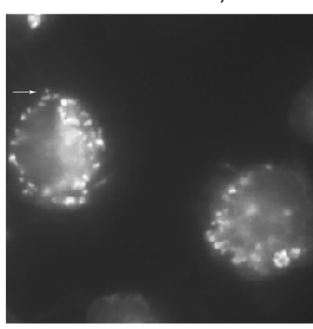

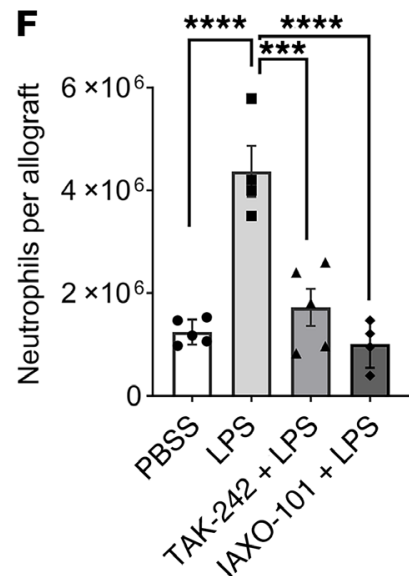

Figure 7. TRAMs exposed to ischemia/reperfusion have increased TLR4 transcription and translocation to the plasma membrane. (A) Heatmap of selected TLR4 pathway genes expressed in murine donor TRAMs exposed to ischemia/reperfusion ( $n=3-5)$. (B) TIr4 and Cd14 mRNA expression in TRAMs harvested from BALF before and after ischemia at 24 hours was assessed by qPCR $(n=3)$. (C) Expression level of TIr4 mRNA in TRAMs harvested from BALF before ischemia and 1 hour, 4 hours, and 24 hours after ischemia, assessed by qPCR $(n=3-5)$. (D) Protein expression of TLR4 at the plasma membrane in TRAMs harvested as in C, assessed by flow cytometry $(n=3-5)$. (E) Immunocytochemistry for TLR4 of TRAMs harvested as described in C. (F) WT C57BL/ 6 donor mice were treated with low-dose i.t. LPS (0.5 $\mu \mathrm{g} / \mathrm{g} \mathrm{BW})$ alone or in combination with TLR4 antagonist (TAK-242; $1 \mu \mathrm{g} / \mathrm{g}$ BW, i.v.) or IAXO-101

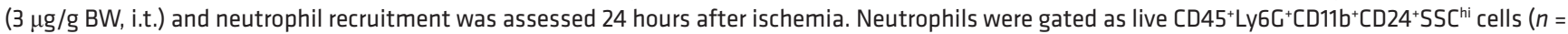
4-5). (B) Graph was analyzed by unpaired $t$ test. (C, D, and F) Graphs were analyzed by 1-way ANOVA followed by Tukey's post hoc test. ${ }^{*} P<0.05$; ${ }^{* *} P<$ $0.01 ;{ }^{* *} P<0.001 ;{ }^{* * *} P<0.0001$. Scale bar: $10 \mu \mathrm{m}$. Arrow points to TLR4 at the plasma membrane.

into the allograft has precluded therapies targeted to the donor or recipient that might attenuate their recruitment and the attendant lung injury. We found that human donor lungs successfully treated for bacterial pneumonia had residual endotoxin despite the clearance of bacterial pathogen. In a mouse lung transplant model, levels of endotoxin insufficient to induce detectable injury in donor lungs was associated with increased neutrophil recruitment as well as physiological markers of allograft injury. This is consistent with the observed association between historic pneumonia in the donor lung and PGD (44).

We used a mouse model of lung transplant to elucidate the mechanisms by which a low level of LPS in the donor lung induces neutrophil recruitment into the allograft. We found that endotoxin in the donor lung primes donor TRAMs to release CXCL2 after lung transplantation, leading to neutrophil recruitment. We also found that ischemia reperfusion injury upregulates both TLR4 and CD14 on TRAMs, potentially creating a feed-forward loop to sustain neutrophil recruitment. The importance of LPS/TLR4/MyD88 signaling in TRAMs was confirmed by using genetic and pharmacologic inhibitors combined with cell-specific depletion and reconstitution studies. In marked contrast to the requirement of NCMs for neutrophil recruitment into allografts from naive donors (13), TRAMs in the donor lung were indispensable and NCMs were dispensable for neutrophil recruitment in LPS-contaminated donors. These findings show that the exposure history in the donor can reshape neutrophil responses during lung transplantation.

Release of ROS during ischemia/reperfusion injury contributes to the pathogenesis of organ dysfunction (37). We analyzed human donor lungs longitudinally to evaluate for the production of ROS. We found that ROS started to increase during the warmischemia phase and continue to do so following reperfusion, but the increase in TLR4 transcription was not observed until 30 minutes 

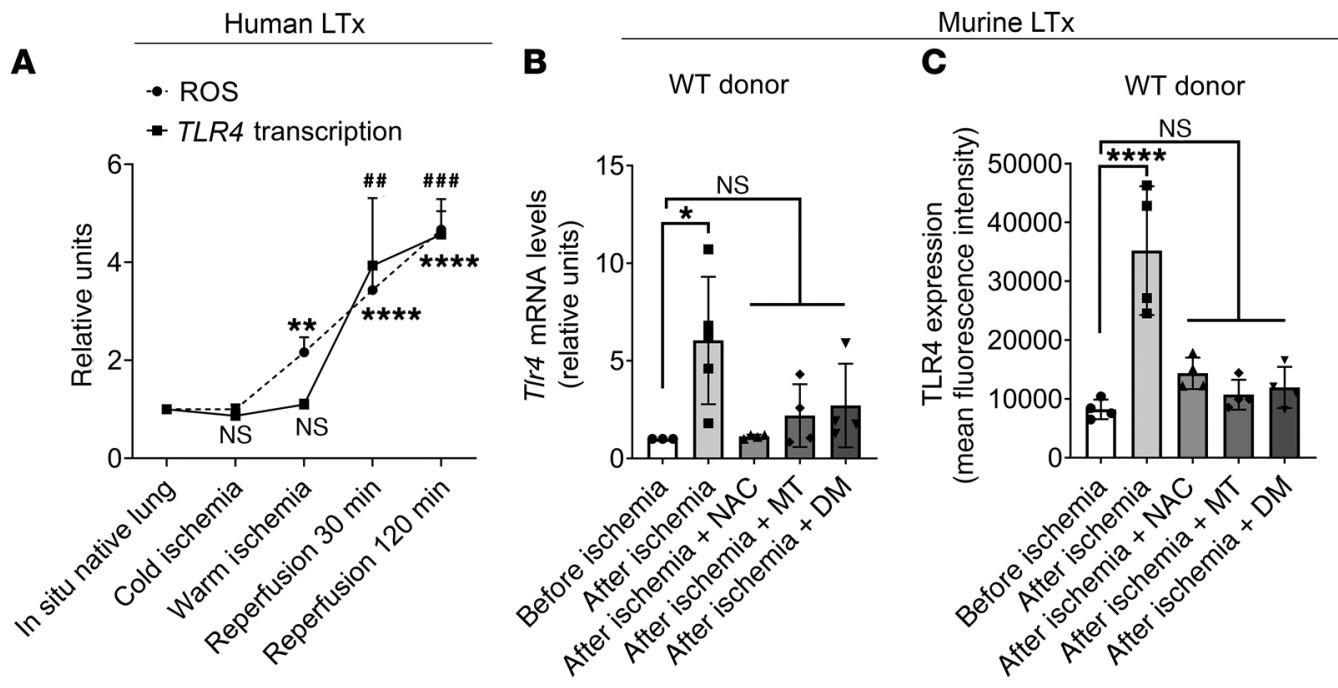

Murine LTX
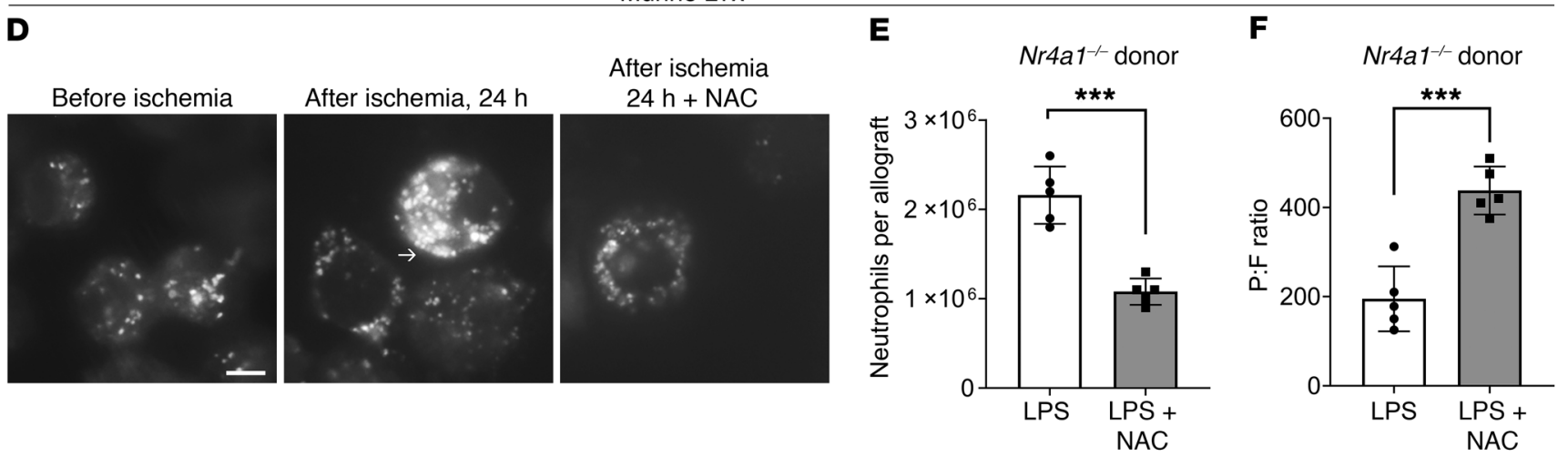

Figure 8. ROS produced during ischemia reperfusion are responsible for TLR4 upregulation. (A) Human donor lungs being used for clinical transplantation were serially biopsied during the different phases of ischemia/reperfusion and development of ROS, and TLR4 transcription of flow-sorted donor TRAMs was determined. (B) WT BALB/c mice were transplanted with WT C57BL/6 donor lungs. In order to test the effect of ROS on TLR4 expression in TRAMs, we treated donors and recipient with NAC (100 mg/kg BW), MT ( $5 \mathrm{mg} / \mathrm{kg} \mathrm{BW,} \mathrm{i.p.),} \mathrm{or} \mathrm{DM} \mathrm{(10} \mathrm{mg/kg} \mathrm{BW,} \mathrm{i.v.),} \mathrm{before} \mathrm{and} \mathrm{after} \mathrm{reperfusion.} \mathrm{Tlr4}$ mRNA levels were assessed 24 hours after ischemia by qPCR in TRAMs harvested from BALF before and after transplantation ( $n=3-5)$. (C) Protein expression of TLR4 at the plasma membrane in TRAMs harvested as in B, assessed by flow cytometry $(n=4)$. (D) Immunocytochemistry for TLR4 of TRAMs harvested as described in B. Nr4a1 -1- donor lungs, which do not develop PGD, were administered low-dose LPS and transplanted into WT recipients with or without NAC treatment. (E) Neutrophil infiltration was determined using flow cytometry and (F) lung function was determined using blood gases and P/F ratio. (B, C) Graphs were analyzed by 1-way ANOVA followed by Tukey's post hoc test. (E, F) Graphs were analyzed by unpaired $t$ test. ${ }^{*} P<0.05$; ${ }^{\# \#}$ and ${ }^{* *} P$ $<0.01$; \#\# and ${ }^{* *} P<0.001 ;{ }^{* * *} P<0.0001$. (A) ${ }^{*}$ ROS; \#TLR4 transcription. Scale bar: $10 \mu \mathrm{m}$. Arrow points to TLR4 at the plasma membrane.

after reperfusion (45). As warm ischemia lasts 45 to 60 minutes, we concluded that the increase in TLR 4 transcription lags generation of ROS by 1 to 2 hours. We confirmed these findings in the murine lung transplant model where the expression of TLR4 increased after transplant and were inhibited using the general ROS scavenger N-acetylcysteine (NAC) (46) as well as the mitochondrial ROS scavengers Mito-Tempo (MT) (39) and dimethyl malonate (DM) (40). Further work is needed to determine the mechanisms by which ROS augment Tlr4 and Cd14 transcription and whether ischemia reperfusion driven ROS also augment TLR4 translocation to the plasma membrane via Src-dependent signaling through generation of ceramide as has been described $(35,47)$.

Our findings provide a mechanistic explanation for the marked and unpredictable heterogeneity between transplant recipients in the incidence and severity of PGD with implications for therapy. Specifically, we found that both primed alveolar macrophages from LPS treated donors and NCMs responded to lung transplant mediated ischemia reperfusion injury by releasing CXCL2, also known as MIP-2 $(13,48)$. While other cytokines and chemokines play a role in the initiation and propagation of the inflammatory response, the focus of our work was on neutrophil recruitment. Our focus on CXCL2 was justified based on our prior publication (13) which revealed a nonredundant role of CXCL2 in neutrophil recruitment to the allograft following lung transplantation and supported by our analysis of several other cytokines in LPS containing donor lungs (Supplemental Figure 1). Causal evidence comes from the murine transplant model where genetic or pharmacological neutralization of CXCL2 in the LPS primed donor lung reduced neutrophil recruitment. Furthermore, we found that inhibition of mitochondrial ROS, which have been implicated in signaling pathways induced by ischemia reperfusion injury (36, 37), attenuated CXCL2 mediated neutrophil recruitment after 
lung transplantation. While removing LPS from donor lungs is unlikely to be feasible, treatment of donor lungs with a history of pneumonia with these inhibitors before transplantation might safely reduce the risk of PGD and expand the pool of lungs available for transplantation. Importantly, we were able to confirm key aspects of our murine model in homologous cell populations isolated from patients with severe pneumonia in our intensive care unit and in patients undergoing lung transplantation in our center. More careful phenotyping of the exposure history in the donor lung might reveal additional environmental exposures that drive PGD. Putative exposures can be causally examined in preclinical murine lung transplant models as we show in this study. While our data using bone marrow chimeric mice combined with scRNA-Seq data implicated TRAMs as the predominant cell type necessary for the production of CXCL2, a role for another cell type in the donor lung releasing CXCL2 should be recognized since it has been shown that alveolar epithelial cells can produce CXCL2 in response to pathogens (49). Additionally, while the focus of our study was on neutrophil recruitment that was dependent on CXCL2, it should be emphasized that other cytokines and chemokines would be necessary for the propagation of downstream inflammatory cascade and need to be further elucidated.

Fulminant bacterial infections or high levels of endotoxin will likely lead to severe injury to the donor lungs, making them unsuitable for clinical transplantation. Our rationale for using low levels of endotoxin was to specifically test the hypothesis that residual PAMPs, which persist after treatment of donor pneumonias and are insufficient to cause injury in the native lungs, can drive the pathogenesis of PGD. We recognize that damageassociated molecular patterns (DAMPs), such as HMGB1, might be released during the ischemia/reperfusion phase and trigger TLR4 activation $(50,51)$. While we tested the role of endotoxin in this study, it is possible that these DAMPs may also contribute to the clinical heterogeneity observed in the incidence of PGD. Hence their role should be explored in future studies. Our current and previously published data suggest the development of PGD involves several cell populations including NCMs, neutrophils, alveolar macrophages, and CMs. The complex signaling pathways leading to their orchestrated sequential recruitment and activation are incompletely understood. Additionally, whether compartmental separation of these DAMPs and PAMPs can selectively activate donor NCMs or TRAMs should also be explored. It is intriguing that NCMs play a role in neutrophil recruitment in response to DAMPs while recipient CMs permeabilize the pulmonary endothelium to promote neutrophil extravasation $(11-13,15)$. In contrast, we found that donor TRAMs are capable of driving both neutrophil recruitment as well as neutrophil extravasation since depletion of TRAMs resulted in a marked decrease in both. Whether TRAMs mediate neutrophil extravasation through the recruitment of recipient classical monocytes remains unknown. Future studies might examine the role of TRAMs in monocyte trafficking to the lung allografts as they might introduce common therapeutic targets to ameliorate both ischemia/reperfusion and endotoxin-associated lung allograft injury.

Our study suggests several avenues for future investigation. While the data using bone marrow chimeric mice combined with scRNA-Seq data implicated TRAMs as the predominant cell type necessary for the production of CXCL2, it has been shown that alveolar epithelial cells can also produce CXCL2 in response to pathogens (49). Additionally, we focused on neutrophil recruitment mediated by CXCL2 as it is the earliest event in the development of PGD. However, our current and previously published data suggest that the development of PGD involves several cell populations including NCMs, neutrophils, alveolar macrophages, and CMs. The complex signaling pathways leading to their orchestrated sequential recruitment and activation are incompletely understood. While we show a clear role for retained endotoxin from different bacterial pathogens in neutrophil recruitment after transplant, our findings do not exclude the possibility that DAMPs, such as HMGB1, might be released during the ischemia/reperfusion phase and also trigger TLR activation $(50,51)$. Finally, the lung microbiome did not reveal significant differences between the pathogenic and nonpathogenic bacteria, making it less likely to account for PGD outcomes. Furthermore, these organisms would likely be cleared by the broad-spectrum antibiotics that are administered routinely to the donor before transplant and the recipient after transplant.

In conclusion, we show that contamination of donor lungs with levels of LPS insufficient to induce detectable injury before transplantation induces TLR4/MyD88 signaling in ischemia/ reperfusion-primed TRAMs to release CXCL2, leading to neutrophil recruitment. This priming of donor TRAMs is independent of the requirement for NCMs to recruit neutrophils to lung allografts from naive donors after lung transplantation. Our results highlight the potential utility of strategies targeting CXCL2, scavenging ROS, or blocking TLR4 or coreceptor CD14 on donor TRAMs and NCMs to reduce neutrophil recruitment and PGD in endotoxin-containing donor lungs.

\section{Methods}

\section{Human samples}

Transplant patients. Bronchoscopic bronchoalveolar lavage and lung biopsies were used to collect donor TRAMs at the different time points outlined in the experiments.

P. aeruginosa-infected patients. Small aliquots (1-4 mL) of nonbronchoscopic bronchoalveolar lavage (NBBAL) fluid were collected from patients with suspected infection (16).

$B A L F$ processing. Fluid was filtered through a $40-\mu$ m nylon cell strainer and following staining, cells were sorted at the Northwestern University Robert H. Lurie Comprehensive Cancer Center Flow Cytometry Core facility using FACSARIA III (BD Bioscience). TRAMs were identified using sequential gating as singlets $/ \mathrm{CD}^{4} 5^{+} /$live $/ \mathrm{CD} 15^{-} /$

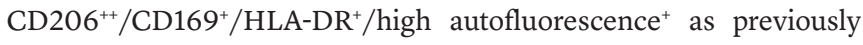
described (16).

\section{Mice and procedures}

WT C57BL/6J (B6), B6.FVB-Tg(ITGAMDTR/EGFP)-34Lan/J (CD11b-DTR), and $\mathrm{Rag}^{-/} \mathrm{IL} 2 \mathrm{~g}^{-/-}$mice with transgenes for human GM-CSF and $I L-3$ (MISTRG mice) (16), $\mathrm{CxCr}^{-/-}$, and $\mathrm{Nr}^{-11^{-/-}}$mice were obtained from the Jackson Laboratory. Tlr $4^{-/-}$and $C d 11 c^{\text {Cre }}$ $M y d 88^{4}$ mice in B6 background were a gift from Harris Perlman (Department of Medicine, Northwestern University, Chicago) and B6 lysozyme $M$ (LysM)-GFP mice were originally obtained from 


\begin{tabular}{|c|c|c|c|}
\hline Antigen & Clone & Fluorochrome & Manufacturer \\
\hline CD24 & M1/69 & BUV395/APC & BD Horizon \\
\hline CD45 & 30-F11 & FITC/APC & BioLegend \\
\hline CD11b & M1/70 & FITC/APCCy7 & BD Horizon/BioLegend \\
\hline $\mathrm{I}-\mathrm{A} / \mathrm{I}-\mathrm{E}$ & M5/114.15.2 & FITC/PercpCy5.5 & BioLegend \\
\hline Ly6C & HK 1.4 & efluor450 & eBioscience \\
\hline Ly6C & 1A8 & AlexaFluor 700 & BioLegend \\
\hline NK1.1 & PK136 & AlexaFluor 700 & BD Biosciences \\
\hline CD64 & X54-5/7.1 & $\mathrm{PE}$ & BioLegend \\
\hline SiglecF & E50-2440 & PECF594 & BD Biosciences \\
\hline CD11c & HL3 & PECy7 & BD Biosciences \\
\hline TLR4 & MT5510 & PECy7 & BioLegend \\
\hline
\end{tabular}

Klaus Ley (La Jolla Institute for Immunology). All mice were maintained in a specific pathogen-free facility at the Center for Comparative Medicine at Northwestern University and used for the described experiments at the age of 9 to 14 weeks and between 24 and $28 \mathrm{~g}$ body weight.

LPS induced lung injury. LPS from E. coli (serotype O111:B4; MilliporeSigma), P. aeruginosa 10 (serotype 10.22; MilliporeSigma), and Klebsiella pneumoniae (source strain ATCC 15380; MilliporeSigma) were used at $2 \mu \mathrm{g} / \mathrm{g} \mathrm{BW}$ (body weight) or $0.5 \mu \mathrm{g} / \mathrm{g} \mathrm{BW}$ (E. coli) and $0.2 \mu \mathrm{g} / \mathrm{g}$ BW P. aeruginosa and K. pneumoniae diluted in $50 \mu \mathrm{L}$ PBS and instilled i.t. MISTRG mice reconstitution and mouse pneumonia model were previously described (16).

Broncho alveolar lavage fluid. BALF was obtained by instilling lung airways 5 times with $0.5 \mathrm{~mL}$ PBS. BALF was centrifuged and the supernatant was used for chemokine and cytokine determination as described below.

Purified anti-CXCL2 (clone 40605; R\&D Systems) or rat IgG2B isotype control (R\&D Systems), TAK-242, TLR4 antagonist (AbMole), IAXO-101, CD14/TLR4 antagonist (Innaxon), NAC (MilliporeSigma), MT (MilliporeSigma), and DM (MilliporeSigma) were commercially acquired. Anti-CXCL2 antibodies or isotype control (200 $\mu \mathrm{g} / \mathrm{ms}$, i.v.), TAK-242 (1 $\mu \mathrm{g} / \mathrm{g}$ BW, i.v.), and IAXO-101 (3 $\mu \mathrm{g} / \mathrm{g}$ BW, i.t.) were administrated to the donors 30 minutes before i.t. LPS $(52,53)$. For ROS scavenger experiments, donor mice were treated with the first dose of the NAC (100 mg/kg BW, i.v.), MT ( $5 \mathrm{mg} / \mathrm{kg} / \mathrm{ms}$, i.p.), or DM $(10 \mathrm{mg} / \mathrm{kg}$ BW, i.v.) 1 hour before transplant; then, recipient mice received same doses of ROS scavenger 5 minutes before reperfusion 1 hour (NAC) or 2 hours (TM and DM) after reperfusion.

Mouse lung transplant with and without LPS. Orthotopic murine left lung transplantation was performed as previously described (13). Briefly, a donor mouse was anesthetized with a mixture of xylazine $(10 \mathrm{mg} / \mathrm{kg})$ and ketamine $(100 \mathrm{mg} / \mathrm{kg})$. Donor lungs were flushed through the pulmonary artery with $3 \mathrm{~mL}$ saline solution and the heartlung block was excised and kept in cooled $\left(4^{\circ} \mathrm{C}\right)$ preservative solution. The bronchus, pulmonary vein, and artery were dissected and prepared for anastomosis. A customized cuff made of a Teflon intravenous catheter was applied to the vascular structures and fixated with a 10-0 nylon ligature. After placement of a microvessel clip on the bronchus to avoid airway infiltration with preservative solution, the graft was stored at $4^{\circ} \mathrm{C}$ for a period of 90-120 minutes of coldischemic time before implantation. The recipient mouse received subcutaneous buprenorphine $(0.1 \mathrm{mg} / \mathrm{kg}) 30$ minutes before the thoracic surgical incision and every 6 hours as needed after the transplant procedure. The recipient mouse was intubated and a left-sided thoracotomy was performed within the third intercostal space. The recipient's native lung was gently clamped and pulled out of the thoracic cavity. The space between the artery, the vein, and the bronchus was dissected separately. The artery and vein were temporarily occluded using 8-0 nylon ligatures. The anastomoses were completed by fixating each cuff with 10-0 nylon ligatures. The 8-0 ligatures were released (first vein, then artery) and the lung inflated. The chest incision was closed and recipients separated from the ventilator when spontaneous respiration resumed. No antibiotics or immunosuppressive agents were used postoperatively in any groups. For LPS-treated donors with or without TLR4 or CD14 antagonist, we treated donor mice 30 minutes before procurement with $0.5 \mu \mathrm{g} / \mathrm{g} / \mathrm{BW}$ i.t. LPS with or without $100 \mu \mathrm{g} / \mathrm{ms} /$ i.v., respectively.

Adoptive transfer of TRAMs. A tracheostomy was performed in the donor mice to harvest TRAMs from BALF. Briefly, BALF was obtained with 2 mM EDTA solution in PBS lavage following by centrifugation and red blood cell (RBC) lysis. TRAMs obtained from BAL were resuspended in the final volume for adoptive transfer to the recipient mice $5 \times 10^{5}$ per recipient.

TRAM and monocyte depletion. Clodronate-loaded liposomes and control phosphate-buffered saline liposomes were purchased from Clodronateliposomes.com. At specific time points, mice were injected i.t. $(50 \mu \mathrm{L})$ or i.v. $(200 \mu \mathrm{L})$ with clodronate-loaded liposomes or control PBS-loaded liposomes to deplete macrophages or Ly6C $\mathrm{C}^{\text {hi }}$ monocytes, respectively (54). Cytotoxic anti-CCR2 antibodies were a gift of Steffen Jung (Weizmann Institute of Science, Rehovot, Israel) and used for selective depletion of CCR $2^{+}$classical monocytes as previously described. Diphtheria toxin (List Biologic Laboratories, Inc.) was dissolved in $100 \mu \mathrm{L}$ PBS and administered i.p. to CD11b-DTR mice 24 hours before LPS administration to systematically deplete CD11b-expressing cells.

\section{Compartmental lung intravenous staining for neutrophils extravasation}

Intravenous and intratracheal procedures were performed using an adaptation of previously described methods (34). Briefly, $6 \mu \mathrm{g}$ APC-conjugated anti-CD45 in $100 \mu \mathrm{L}$ sterile PBS was injected i.v. and allowed to circulate for 3 minutes before euthanasia with an overdose of Euthasol (Virbac). Tracheostomy was performed. The vena cava was transected and the right ventricle flushed with $10 \mathrm{~mL}$ HBSS to wash unbound i.v. antibody.

\section{Multicolor flow cytometry}

Single-cell suspensions from the whole lung of the mouse were obtained and murine lung cell staining performed as previously described $(32,55)$. Table 1 shows all antibodies used. Fixed samples were run on a custom LSRFortessa Cell Analyzer (BD Bioscience) or BD FACS Symphony A5-Laser Analyzer for flow cytometry analysis at the Northwestern University Robert H. Lurie Comprehensive Cancer Center Flow Cytometry Core facility. Acquired data were analyzed with FlowJo v10.6 (FlowJo). For TRAM sorting, lung airways were lavaged 5 times with $0.5 \mathrm{~mL}$ PBS. BALF was cen- 
trifuged and the cell pellet was processed and prepared for flow sorting using a FACSARIA 4-Laser Sorter at the Northwestern University Robert H. Lurie Comprehensive Cancer Center Flow Cytometry Core facility.

\section{Wet/dry ratio}

Weight ratio for pulmonary edema was measured by harvesting lungs after reperfusion at defined experiments, weighed, and then placed at $54^{\circ} \mathrm{C}$ until a stable dry weight was achieved. The ratio of wet weight to dry weight was calculated as an indicator of pulmonary edema.

\section{Histology and H\&E staining}

For histological analyses, the whole lung was harvested and gently flushed through the pulmonary artery with $3 \mathrm{~mL}$ saline. Paraformaldehyde (4\%) was instilled into the trachea with a pressure of 10 $\mathrm{cmH}_{2} \mathrm{O}$ then fixed for 48 to 72 hours before being embedded in paraffin. The whole lung was serially sectioned and stained with H\&E. Pictures were acquired using a Nikon TE-2000-U microscope.

\section{ELISA}

Endotoxin (Thermo Fisher Scientific), CXCL1, CXCL2, CXCL10, and TNF- $\alpha$ ELISA was performed using a commercially available kit accordingly to the manufacturer's instructions (R\&D Systems).

\section{Secondary transcriptomic analysis}

RNA-sequencing. RNA isolation and sequencing procedures are detailed in our previous manuscript (34). For all analyses, normalized tables of counts were generated from raw counts using the EdgR package (version 3.18.1). Filtering for low counts was performed by excluding genes that have the expression from more than half of all the samples. Functional enrichment analysis with GO biological processes was performed with GOrilla with the top 1000 genes upregulated in $P$. aeruginosa positive samples for all clusters. Significantly upregulated genes were used to identify the top biological processes $(-\log 10[P$ value]). Heatmaps of pathway genes based on the top GO processes were identified and hierarchical clustering was generated to show the expression of these genes in naive, low, and high virulence samples for MISTRG mice (16).

Reanalysis of single-cell RNA-Seq data sets. To assess expression pattern of TRL 4 in human lung, we queried 3 recently published singlecell RNA-Seq data sets $(29,30,31)$. Data and author's annotations were accessed from GSE122960 (29), tissuestabilitycellatlas.org (30), and GSE135893 (31). Only nondiseased lungs (indicated as donor or control) were used for analysis. Precomputed R objects were loaded into Seurat 3.1.4 and expression of TLR4 was visualized using FeaturePlot command.

\section{Two-photon microscopy}

A custom-built 2-photon microscope running ImageWarp acquisition software (A\&B Software) was used for time-lapse imaging. Mice were anesthetized with an i.p. injection of ketamine $(50 \mathrm{mg} / \mathrm{kg})$ and xylazine $(10 \mathrm{mg} / \mathrm{kg})$ and maintained with administration of half doses every hour through the imaging process. Mice were intubated orotracheally and ventilated with room air at a rate of 120 breaths/ min and with a tidal volume of $0.5 \mathrm{~mL}$. Left thoracotomy was performed to expose the left lung, and the lung was imaged using a custom-built chamber maintained at $37^{\circ} \mathrm{C}$. VetBond ring was used to attach the lung tissue to the bottom of the cover glass without direct pressure on the exposed lung. For time-lapse imaging of leukocyte migration in the tissue parenchyma, we averaged 15 video-rate frames $(0.5 \mathrm{~s} / \mathrm{slice})$ during the acquisition to match the ventilator rate and minimize movement artifacts. Each plane represents an image of $220 \times 240 \mu \mathrm{m}$ in the $x$ and $y$ dimensions. To visualize blood vessels, $20 \mu \mathrm{L}$ of 655-nm nontargeted Quantum dots (Q-dots) in 100 $\mu \mathrm{L}$ PBS was injected i.v. before imaging. Two-photon excitation produces a second harmonic signal from collagen (56) around alveoli, thus providing a useful landmark for the air spaces. Explanted tissue was examined as described (56-58).

\section{mRNA isolation and qPCR}

Total RNA was extracted using miRNeasy Mini Kit (Qiagen). cDNA was synthesized from $1 \mu \mathrm{g}$ total RNA by using a qScript cDNA Synthesis kit (Quanta Biosciences) and mRNA expression was determined by qPCR using iTaq Universal Probes Supermix (Bio-Rad). Relative expression of the transcripts was determined according to the $\Delta \Delta \mathrm{Ct}$ method using actin or RPL19 as reference for normalization. Primers used were as follows: cxcl2-F, CCCAGACAGAAGTCATAGCCAC; cxcl2-R, GCCTTGCCTTTGTTCAGTATC; tlr4-F, TGGCTGGTTTACACATCCATCGGT; tlr4-R, TGGCACCATTGAAGCTGAGGTCTA; B-actin-F, CACCACACCTTCTACAATGA; B-actin-R, GTCTCAAACATGATCTGGGT.

\section{Reactive oxygen species measurement}

The ROS levels were examined in lung samples using a commercially available assay (In Vitro OxiSelect Assay Kit, Cell Biolabs). Briefly, we homogenized the lung tissue obtained at the different time points during the lung transplantation (native donor lung before reperfusion, cold ischemia, warm ischemia, 30 minutes after reperfusion) according to the manufacturer's recommendation and resuspended it in the assay buffer. The cell-permeable fluorogenic probe was then used to assess the ROS levels. Fluorescence was read on a Spectramax plate reader.

\section{Immunocytochemistry and fluorescence microscopy}

We used cytospin to immobilize 10,000-50,000 TRAMs onto slides, where cells were fixed with $4 \%$ formaldehyde, permeabilized with $0.5 \%$ Triton X-100, and immunostained with anti-PE-TLR4 antibody (BioLegend, clone HTA125). Nuclei were stained with Hoechst 33342 (MilliporeSigma). For immunocytochemistry, cells were imaged using a Zeiss Axioplan 2 fluorescence microscope. Confocal images were acquired using a Zeiss Axio Imager Z2 with ApoTome.2 microscope equipped with Axiocam 503 Mono, X-Cite 120 LED Boost System, and Zen 2.3 software (Carl Zeiss).

\section{Microbiome analysis}

Ten BAL washings of donor lungs were obtained in the operating room at the time of organ implantation. Rectal swabs were obtained for 5 of these patients at the same time. Samples were immediately centrifuged to collect cellular DNA and stored at $-80^{\circ} \mathrm{C}$. Samples then underwent batched genomic DNA extraction, PCR amplification targeting the 16S ribosomal RNA gene, pooling, and high-throughput sequencing with paired-end, 300-base reads on the Illumina MiSeq platform to obtain overall taxonomic compositions of the microbial communities. A sterile swab obtained just before DNA extraction 
was used for the negative control. Distances for each sample's overall microbial profile were generated via square root transformation of the relative abundances of each taxon. A nonmetric multidimensional scaling plot was created by using Bray-Curtis dissimilarity through PRIMER version 7 as shown in Supplemental Figure 2. 2D stress was 0.14. An analysis of similarity (ANOSIM) was used to determine statistical similarities among groups.

\section{TRAM viability in vitro}

TRAM viability was measured with acridine orange/propidium iodide (AO/PI) nucleic acid binding dyes method, using a Nexcelom cellometer. AO is cell permeable; all stained nucleated cells generate a green fluorescence and PI only enters cells with compromised membranes. Therefore, dying, dead, and necrotic nucleated cells stained with PI generate a red fluorescence. Cell viability was calculated by examining the ratio of the number of live to the number of dead fluorescing cells (Nexcelom).

\section{Statistics}

Data analysis was performed using Prism 8 (GraphPad Software). Results are expressed as mean \pm SD and the $n$ values for each data set are provided in the figure legends. Statistical significance was assessed by 2-tailed Student's $t$ test or 1-way ANOVA followed by Tukey's post hoc test. A $P$ value less than 0.05 was considered significant.

\section{Study approval}

All procedures were approved by The Institutional Animal Care and Use Committee (ISO0002248) at Northwestern University. Animals received humane care in compliance with the NIH Guide for the Care and Use of Laboratory Animals (National Academies Press, 2011) and the principles of laboratory animal care formulated by the National Society for Medical Research. Human protocol was approved by the Northwestern University Institutional Review
Board (STU00106589 and STU00201397). All study subjects provided informed written consent.

\section{Author contributions}

MA and EL contributed to conceptualization, study design and methodology, data collection and formal analysis, and manuscript writing. SFC, RF, NSC, TM, and JMW contributed to formal analysis, methodology, and manuscript drafting. QW, HS, SR, WL, and $\mathrm{AH}$ conducted experiments. NJ and ZR contributed to formal analysis and methodology. DK, MQ, AVM, FLNS, SCR, and CK contributed to methodology, validation, formal analysis, and visualization. GRSB and AB contributed to conceptualization, methodology, validation, formal analysis, investigation, resources, data curation, writing, visualization, supervision, project administration, and funding acquisition.

\section{Acknowledgments}

This work was supported by NIH grants HL145478, HL147290, and HL147575 (to AB), NIH grants P01 AG049665 and P01 HL071643 and Department of the Army grant W81XWH-15-1-0215 (to GRSB and AVM), and NIH grants 5P01AG049665 and 5P01HL071643 (to NSC). The Northwestern University Flow Cytometry Core Facility is supported by a Cancer Center Support Grant (NCI CA060553). We thank Suchitra Swaminathan, director of the flow cytometry core facility, for providing professional technical assistance and Elena Susan for administrative assistance and submission of the manuscript.

Address correspondence to: Ankit Bharat, Northwestern University Feinberg School of Medicine, Divisions of Thoracic Surgery, Pulmonary and Critical Care Medicine, 676 N. Saint Clair Street, Suite 650, Chicago, Illinois, 60611 USA. Phone: 312.926.7552; Email: abharat@nm.org.
1. Charlson ES, et al. Topographical continuity of bacterial populations in the healthy human respiratory tract. Am J Respir Crit Care Med. 2011;184(8):957-963.

2. Huang YJ, Charlson ES, Collman RG, Colombini-Hatch S, Martinez FD, Senior RM. The role of the lung microbiome in health and disease. A National Heart, Lung, and Blood Institute workshop report. Am J Respir Crit Care Med. 2013;187(12):1382-1387.

3. Ruiz I, et al. Donor-to-host transmission of bacterial and fungal infections in lung transplantation. Am J Transplant. 2006;6(1):178-182.

4. Abraham E. Neutrophils and acute lung injury. Crit Care Med. 2003;31(4 Suppl):S195-S199.

5. Puljic R, et al. Lipopolysaccharide-induced lung inflammation is inhibited by neutralization of GM-CSF. Eur JPharmacol. 2007;557(2-3):230-235.

6. Chabot F, Mitchell JA, Gutteridge JM, Evans TW. Reactive oxygen species in acute lung injury. Eur Respir J. 1998;11(3):745-757.

7. Saffarzadeh M, et al. Neutrophil extracellular traps directly induce epithelial and endothelial cell death: a predominant role of histones. PLOS One. 2012;7(2):e32366.

8. Narasaraju T, et al. Excessive neutrophils and neutrophil extracellular traps contribute to acute lung injury of influenza pneumonitis. Am J Pathol. 2011;179(1):199-210.

9. Williams AE, Chambers RC. The mercurial nature of neutrophils: still an enigma in ARDS? Am JPhysiol Lung Cell Mol Physiol. 2014;306(3):L217-L230.

10. Kreisel D, et al. Bcl3 prevents acute inflammatory lung injury in mice by restraining emergency granulopoiesis. J Clin Invest. 2011;121(1):265-276.

11. Bharat A, Kreisel D. Immunopathogenesis of primary graft dysfunction after lung transplantation. Ann Thorac Surg. 2018;105(3):671-674.

12. Hsiao HM, et al. Spleen-derived classical monocytes mediate lung ischemia-reperfusion injury through IL-1 $\beta$. J Clin Invest. 2018;128(7):2833-2847.

13. Zheng $\mathrm{Z}$, et al. Donor pulmonary intravascular nonclassical monocytes recruit recipient neutrophils and mediate primary lung allograft dysfunction. Sci Transl Med. 2017;9(394):eaal4508.

14. Chignard M, Balloy V. Neutrophil recruitment and increased permeability during acute lung injury induced by lipopolysaccharide. Am J Physiol Lung Cell Mol Physiol. 2000;279(6):L1083-L1090.

15. Bharat A, McQuattie-Pimentel AC, Budinger GRS. Non-classical monocytes in tissue injury and cancer. Oncotarget. 2017;8(63):106171-106172.

16. Walter JM, et al. Multidimensional assessment of the host response in mechanically ventilated patients with suspected pneumonia. Am J Respir Crit Care Med. 2019;199(10):1225-1237.

17. Maus UA, et al. Monocytes are potent facilitators of alveolar neutrophil emigration during lung inflammation: role of the CCL2-CCR2 axis. J Immunol. 2003;170(6):3273-3278.

18. Dhaliwal K, et al. Monocytes control second-phase neutrophil emigration in established lipopolysaccharide-induced murine lung injury. Am J Respir Crit Care Med. 2012;186(6):514-524.

19. Beck-Schimmer B, Schwendener R, Pasch T, Reyes L, Booy C, Schimmer RC. Alveolar macrophages regulate neutrophil recruitment in endotoxin-induced lung injury. Respir Res. 2005;6:61.

20. Bhatia M, Zemans RL, Jeyaseelan S. Role of chemokines in the pathogenesis of acute lung injury. Am J Respir Cell Mol Biol. 2012;46(5):566-572.

21. Becker J, Poroyko V, Bhorade S. The lung microbiome after lung transplantation. Expert Rev Respir Med. 2014;8(2):221-231.

22. Mitchell AB, Glanville AR. The human respiratory microbiome: implications and impact. Semin 
Respir Crit Care Med. 2018;39(2):199-212.

23. Wu Q, et al. Gut microbiota can impact chronic murine lung allograft rejection. Am J Respir Cell Mol Biol. 2019;60(1):131-134

24. Guo Y, et al. Vendor-specific microbiome controls both acute and chronic murine lung allograft rejection by altering $\mathrm{CD}^{+}{ }^{+} \mathrm{Foxp}^{+}$regulatory $\mathrm{T}$ cell levels. Am J Transplant. 2019;19(10):2705-2718.

25. Moffatt MF, Cookson WO. The lung microbiome in health and disease. Clin Med (Lond). 2017;17(6):525-529.

26. Tsai MH, Liu YY, Chen CC. OutbreakFinder: a visualization tool for rapid detection of bacterial strain clusters based on optimized multidimensional scaling. PeerJ. 2019;7:e7600.

27. Grommes J, Soehnlein O. Contribution of neutrophils to acute lung injury. Mol Med. 2011;17(34):293-307.

28. Faller S, et al. Hydrogen sulfide limits neutrophil transmigration, inflammation, and oxidative burst in lipopolysaccharide-induced acute lung injury. Sci Rep. 2018;8(1):14676.

29. Reyfman PA, et al. Single-cell transcriptomic analysis of human lung provides insights into the pathobiology of pulmonary fibrosis. Am J Respir Crit Care Med. 2019;199(12):1517-1536.

30. Madissoon E, et al. scRNA-seq assessment of the human lung, spleen, and esophagus tissue stability after cold preservation. Genome Biol. 2019;21(1):1.

31. Habermann AC, et al. Single-cell RNA-sequencing reveals profibrotic roles of distinct epithelial and mesenchymal lineages in pulmonary fibrosis. bioRxiv. https:/www.biorxiv.org/content/10.1101/753806v1. Published September 6, 2019. Accessed June 3, 2020.

32. Bharat A, et al. Flow cytometry reveals similarities between lung macrophages in humans and mice. Am J Respir Cell Mol Biol. 2016;54(1):147-149.

33. Miller SI, Ernst RK, Bader MW. LPS, TLR4 and infectious disease diversity. Nat Rev Microbiol. 2005;3(1):36-46.

34. Koch CM, et al. A Beginner's guide to analysis of RNA sequencing data. Am J Respir Cell Mol Biol. 2018;59(2):145-157.

35. Powers KA, et al. Oxidative stress generated by hemorrhagic shock recruits Toll-like receptor 4 to the plasma membrane in macrophages. J Exp Med. 2006;203(8):1951-1961.

36. Prag HA, Kula-Alwar D, Beach TE, Gruszczyk AV Burger N, Murphy MP. In: Sies H ed. Oxidative Stress. Academic Press; 2020:513-38.

37. Wu MY, et al. Current mechanistic concepts in ischemia and reperfusion injury. Cell Physiol Biochem. 2018;46(4):1650-1667.

38. Inci I, et al. Prevention of primary graft dysfunction in lung transplantation by $\mathrm{N}$-acetylcysteine after prolonged cold ischemia. J Heart Lung Transplant. 2010;29(11):1293-1301.

39. Trnka J, Blaikie FH, Smith RA, Murphy MP. A mitochondria-targeted nitroxide is reduced to its hydroxylamine by ubiquinol in mitochondria. Free Radic Biol Med. 2008;44(7):1406-1419.

40. Chouchani ET, et al. Ischaemic accumulation of succinate controls reperfusion injury through mitochondrial ROS. Nature. 2014;515(7527):431-435.

41. Young KA, Dilling DF. The future of lung transplantation. Chest. 2019;155(3):465-473.

42. Somers J, et al. A decade of extended-criteria lung donors in a single center: was it justified? Transpl Int. 2015;28(2):170-179.

43. Courtwright A, Cantu E. Evaluation and management of the potential lung donor. Clin Chest Med. 2017;38(4):751-759.

44. Mojtabaee M, Shahryari S, Sadegh Beigee F. Reasons for donor lungs unsuitability after radiographic selection. Exp Clin Transplant. 2019;17(Suppl 1):120-122.

45. Van Raemdonck DE, Jannis NC, De Leyn PR, Flameng WJ, Lerut TE. Warm ischemic tolerance in collapsed pulmonary grafts is limited to 1 hour. Ann Surg. 1998;228(6):788-796.

46. Geudens N, et al. N-acetyl cysteine pre-treatment attenuates inflammatory changes in the warm ischemic murine lung. J Heart Lung Transplant. 2007;26(12):1326-1332.

47. Tawadros PS, et al. Oxidative stress increases surface Toll-like receptor 4 expression in murine macrophages via ceramide generation. Shock. 2015;44(2):157-165.

48. Frevert CW, Huang S, Danaee H, Paulauskis JD, Kobzik L. Functional characterization of the rat chemokine $\mathrm{KC}$ and its importance in neutrophil recruitment in a rat model of pulmonary inflam- mation. JImmunol. 1995;154(1):335-344.

49. Bello-Irizarry SN, Wang J, Olsen K, Gigliotti F, Wright TW. The alveolar epithelial cell chemokine response to pneumocystis requires adaptor molecule MyD88 and interleukin-1 receptor but not toll-like receptor 2 or 4. Infect Immun. 2012;80(11):3912-3920.

50. Sharma AK, LaPar DJ, Stone ML, Zhao Y, Kron IL, Laubach VE. Receptor for advanced glycation end products (RAGE) on iNKT cells mediates lung ischemia-reperfusion injury. Am J Transplant. 2013;13(9):2255-2267.

51. Paudel YN, Angelopoulou E, Piperi C, Balasubramaniam VRMT, Othman I, Shaikh MF. Enlightening the role of high mobility group box 1 (HMGB1) in inflammation: Updates on receptor signalling. Eur J Pharmacol. 2019;858:172487.

52. Sha T, Sunamoto M, Kitazaki T, Sato J, Ii M, Iizawa Y. Therapeutic effects of TAK-242, a novel selective Toll-like receptor 4 signal transduction inhibitor, in mouse endotoxin shock model. Eur J Pharmacol. 2007;571(2-3):231-239.

53. Haziak K, et al. Effect of CD14/TLR4 antagonist on $\mathrm{GnRH} / \mathrm{LH}$ secretion in ewe during central inflammation induced by intracerebroventricular administration of LPS. J Anim Sci Biotechnol. 2018;9:52.

54. Van Rooijen N, Sanders A. Liposome mediated depletion of macrophages: mechanism of action, preparation of liposomes and applications. J Immunol Methods. 1994;174(1-2):83-93.

55. Misharin AV, et al. Monocyte-derived alveolar macrophages drive lung fibrosis and persist in the lung over the life span. J Exp Med. 2017;214(8):2387-2404.

56. Gelman AE, et al. Cutting edge: Acute lung allograft rejection is independent of secondary lymphoid organs. J Immunol. 2009;182(7):3969-3973.

57. Halle S, et al. Induced bronchus-associated lymphoid tissue serves as a general priming site for $\mathrm{T}$ cells and is maintained by dendritic cells. JExp Med. 2009;206(12):2593-2601.

58 . Kreisel D, et al. In vivo two-photon imaging reveals monocyte-dependent neutrophil extravasation during pulmonary inflammation. Proc Natl Acad Sci USA. 2010;107(42):18073-18078. 